\title{
Dose-Response Relationships between Second-Hand Smoke Exposure and Depressive Symptoms among Adolescents in Guangzhou, China
}

\author{
Jingya Huang ${ }^{1, \dagger}$, Bin $\mathrm{Xu}^{2,+}$, Dan Guo ${ }^{1}$, Ting Jiang ${ }^{2}$, Wei Huang ${ }^{2}$, Guocong Liu ${ }^{2, *}$ \\ and Xiaohua Ye ${ }^{1, *}$ \\ 1 School of Public Health, Guangdong Pharmaceutical University, 283\# Jianghai Dadao, Haizhu District, \\ Guangzhou 510310, China; jingyi4597@hotmail.com (J.H.); guodan446@163.com (D.G.) \\ 2 Health Education Section, Guangzhou Yuexiu Center for Disease Control and Prevention, \\ 23\# Jiaochang West Road, Yuexiu District, Guangzhou 510055, China; xubin6710@163.com (B.X.); \\ jiangting04@126.com (T.J.); yxqhwei@163.com (W.H.) \\ * Correspondence: Lgc3642@126.com (G.L.); smalltomato@163.com (X.Y.); Tel.: +86-020-3405-5355 (X.Y.) \\ + These authors have contributed equally to this work.
}

Received: 5 March 2018; Accepted: 4 May 2018; Published: 14 May 2018

\begin{abstract}
There has been little focus on the possible association between second-hand smoke (SHS) exposure and depressive symptoms among adolescents. Thus, this study aimed to explore the dose-response relationships between SHS exposure and depressive symptoms among adolescents and differentiate these associations in setting-specific exposure and severity-specific outcomes. A cross-sectional study was conducted using a stratified cluster sampling method to obtain a representative sample of high school students in Guangzhou, China. Depressive symptoms were measured using the Center for Epidemiologic Studies Depression Scale. Univariable and multivariable logistic regression models were used to explore the potential associations between SHS exposure and depressive symptoms. Among 3575 nonsmoking students, $29.6 \%$ were classified as having probable depressive symptoms and $9.6 \%$ had severe depressive symptoms. There were monotonically increasing dose-response relationships between setting-specific (public places, homes, or indoor/outdoor campuses) SHS exposure and severity-specific (probable or severe) depressive symptoms. When examining these relations by source of exposure, we also observed similar dose-response relationships for SHS exposure in campuses from smoking teachers and from smoking classmates. Our findings suggest that regular SHS exposure is associated with a significant, dose-dependent increase in risk of depressive symptoms among adolescents, and highlight the need for smoke-free environments to protect the health of adolescents.
\end{abstract}

Keywords: second-hand smoke; smoking; depressive symptoms; adolescents

\section{Introduction}

The Centers for Disease Control and Prevention reported that there is no risk-free level of second-hand smoke (SHS) exposure [1]. The latest retrospective analysis of the worldwide burden of disease attributable to SHS exposure indicated that $40 \%$ of nonsmoking children are exposed to SHS [2]. In China, 740 million (including 180 million children) nonsmokers are exposed to SHS [3]. Global youth tobacco surveillance also reported that nearly half of adolescents are exposed to SHS in homes $(42.5 \%)$ or in public places (55.1\%) [4].

There is increasing evidence suggesting that smoking may cause depressive symptoms or depression [5,6]. It is conceivable that nonsmokers exposed to a high level of SHS may also experience depression as a result. Additionally, growing evidence suggests that SHS may be associated with 
depression through its effects on chronic diseases, chronic stress, and the dopamine system [7-13]. Based on the above findings, we hypothesized that there may be a potential relationship between SHS exposure and depressive symptoms. Epidemiology evidence about the relation between SHS exposure and depressive symptoms has been reported mainly among adults [14-19], and current evidence is inconsistent. A few studies have demonstrated significant relations [14-18], while a nonsignificant relation was found in another report [19]. Compared to the number of studies on adults, few studies report on this relation in adolescents $[20,21]$. Furthermore, there is still no evidence to show the effects of campus SHS exposure on depressive symptoms among adolescents. Therefore, the present study aimed to explore the potential dose-response relationship between SHS exposure and depressive symptoms among adolescents and differentiate this relationship in setting-specific exposure and severity-specific outcomes to make exposure and outcomes clearer.

\section{Methods}

\subsection{Ethics Statement}

The study was approved by the Ethics Committee of Guangdong Pharmaceutical University, and it was performed in accordance with the approved guidelines (2016-2017). This survey was qualified as involving no risks to study participants. The goals of the study were given to the participants and they expressed their willingness to participate. Written informed consents were obtained from parents or guardians of participants.

\subsection{Study Design and Sampling}

The target population was high school students. A cross-sectional study was conducted in Guangzhou, China between March and April 2016. Note that high schools in most parts of China are generally rated as key schools (or prestigious schools) and ordinary schools (or nonprestigious schools) according to education level and teaching quality. Therefore, this study used a stratified cluster sampling process to obtain a representative sample. First, all high schools were divided into two categories (prestigious or nonprestigious schools). Three high schools were randomly sampled from prestigious schools and four high schools were randomly sampled from nonprestigious schools, which were selected with probability proportional to the number of the schools. Second, classes in the selected schools were randomly sampled proportional to the school enrollment size, and all students in the sampled classes were eligible to participate.

After obtaining informed consent, eligible students were asked to complete a face-to-face survey conducted by trained interviewers. A total of 3833 participants were interviewed, of whom 3657 (95.4\%) were willing to participate in this survey. The remaining $176(4.6 \%)$ participants refused to participate since they had to take part in extracurricular training during the investigation. Among 3657 study participants, 3575 (97.8\%, 3575/3657) participants were nonsmokers and $82(2.2 \%, 82 / 3657)$ were smokers.

\subsection{Data Collection and Quality Control}

All interviewers in each school were trained to ensure that the survey was carried out according to the protocol and that operation procedures were identical across all schools. In order to evaluate the feasibility of this investigation, a pilot study was carried out before formal investigation. Two levels of a quality control system (including quality controller and quality leader) were used to check for potential errors in the questionnaires. Data quality was also assured by using double entry procedures to automatically detect data entry errors. Before carrying out statistical analyses, raw data was cleaned to detect and correct (or remove) corrupt or inaccurate records. 


\subsection{Study Variables}

Depressive symptoms-The outcome variable was self-reported depressive symptoms measured by the Chinese version of the Center for Epidemiologic Studies Depression (CES-D) Scale [22], which is widely used in healthy adults and adolescents [23-25]. The 20-item CES-D Scale measures the levels of depressive symptoms experienced in the past week. The scores of CES-D range from 0 to 60 , and the cutoffs of 16 and 25 represent the thresholds for probable depressive symptoms (CES-D $\geq 16$ ) and severe depressive symptoms (CES-D $\geq 25$ ), respectively [26]. Cronbach's alpha for the CES-D scale in this study was 0.854 , suggesting good internal consistency of the questionnaire.

SHS exposure-The main independent variable was self-reported SHS exposure, which was defined as nonsmokers' inhalation of the smoke exhaled from smokers on at least one day a week in the past 7 days [27]. Participants were asked if they had smoked over 100 cigarettes in their lifetime [28-30], and those responding "no" were defined as nonsmokers. The nonsmokers were asked if they had SHS exposure in public places, homes, indoor campuses, and outdoor campuses, respectively. Frequency of SHS exposure was reported as a continuous variable (days/week), and was also categorized into three groups: <1 day/week (no exposure), 1-4 days/week, and 5-7 days/week.

Covariate variables-Covariates including potential mediators and confounders were chosen a priori on the basis of a literature review. Covariates in this study included gender, grade (4-5 or $1-2)$, only child (yes or no), monthly pocket money ( $<¥ 100, ¥ 100-399$, or $\geq ¥ 400)$, prestigious schools (yes or no), parents' education (neither from high school, one from high school, or both from high school), negative life events (yes or no), and disease history $(0,1,2$, or $\geq 3$ ). Negative life events were measured by a response of 'yes' to any of the following events occurring in participants' families in the past month: death of family members, violent/suicidal/criminal behaviors of family members, separation from parents, severe medical problems, accident/disaster, theft, financial problems, or poor housing. For the question of disease history in the past month, participants were asked if they had the following diseases or symptoms: asthma, shortness of breath, frequent coughing, feeling of discomfort in the throat, irritation in eyes, irritation in nose, or other diseases.

\subsection{Statistical Analysis}

All data were entered in duplicate into the EpiData version 3.1 database (The EpiData Association, Odense, Denmark), and then every inconsistency was checked by the consistency test. Univariable and multivariable logistic regression models were used to calculate the odds ratios (ORs) and 95\% confidence interval (CIs) for evaluating the potential relationship between SHS exposure and depressive symptoms. Potential confounders were controlled by a review of putative risk factors for depressive symptoms and a 10\% or greater change in the $\beta$ coefficients for SHS exposure between the crude and the adjusted models. Linear trends of SHS exposure were assessed by modeling exposures as continuous variables (arithmetic or logarithmic scale) or ordinal variables in the logistic models. We defined a two-sided $p$-value of $<0.05$ as being of statistical significance. All statistical analyses were performed using Stata version 14.0 (StataCorp LP, College Station, TX, USA). For this study, only nonsmokers were included in the analyses.

\section{Results}

\subsection{Characteristics of the Sample}

Among 3575 nonsmokers, the mean age was $15.0 \pm 1.8$ years, and $49.1 \%$ were female students. As to depressive symptoms, 1058 (29.6\%) were classified as having probable depressive symptoms and $343(9.6 \%)$ were classified as severe depressive symptoms. SHS exposure was highest in public places $(49.5 \%)$, followed by exposure in homes $(34.5 \%)$, in outdoor campuses $(29.2 \%)$, and in indoor campuses $(22.7 \%)$ (Table 1$)$. 
Table 1. Demographic characteristics of the study participants.

\begin{tabular}{|c|c|c|c|c|c|}
\hline Characteristics & $n$ & $\%$ & Characteristics & $n$ & $\%$ \\
\hline \multicolumn{3}{|c|}{ Probable Depressive symptoms } & \multicolumn{3}{|l|}{ Gender } \\
\hline CES-D score $<16$ & 2517 & 70.4 & Female & 1757 & 49.1 \\
\hline CES-D score $\geq 16$ & 1058 & 29.6 & Male & 1818 & 50.9 \\
\hline \multicolumn{3}{|c|}{ Severe depressive symptoms } & \multicolumn{3}{|l|}{ Monthly pocket money (¥) } \\
\hline CES-D score $<25$ & 3232 & 90.4 & $<100$ & 2039 & 57.0 \\
\hline CES-D score $\geq 25$ & 343 & 9.6 & 100-399 & 1125 & 31.5 \\
\hline \multicolumn{3}{|c|}{ SHS exposure in indoor public places } & $\geq 400$ & 411 & 11.5 \\
\hline No & 1806 & 50.5 & \multicolumn{3}{|l|}{ Parents' education } \\
\hline Yes & 1769 & 49.5 & Neither from high school & 1787 & 50.0 \\
\hline \multicolumn{3}{|l|}{ SHS exposure in homes } & One from high school & 622 & 17.4 \\
\hline No & 2342 & 65.5 & Both from high school & 1166 & 32.6 \\
\hline Yes & 1233 & 34.5 & Prestigious schools & & \\
\hline \multicolumn{3}{|c|}{ SHS exposure in indoor campuses } & No & 1307 & 36.6 \\
\hline No & 2763 & 77.3 & Yes & 2268 & 63.4 \\
\hline Yes & 812 & 22.7 & Grade & & \\
\hline \multicolumn{3}{|c|}{ SHS exposure in outdoor campuses } & $1-2$ & 2329 & 65.2 \\
\hline No & 2532 & 70.8 & $4-5$ & 1246 & 34.8 \\
\hline Yes & 1043 & 29.2 & Only child & & \\
\hline \multicolumn{3}{|l|}{ Disease history } & No & 1353 & 37.8 \\
\hline 0 & 885 & 27.6 & Yes & 2222 & 62.2 \\
\hline 1 & 845 & 26.4 & Negative life events & & \\
\hline 2 & 627 & 19.6 & No & 2179 & 38.2 \\
\hline$\geq 3$ & 845 & 26.4 & Yes & 1345 & 61.8 \\
\hline
\end{tabular}

$n$, number of participants; \%, the proportion of participants surveyed; SHS, second-hand smoke; CES-D, the left for Epidemiologic Studies Depression.

\subsection{Relation between Binary SHS Exposure and Probable Depressive Symptoms}

In contrast to no exposure (Table 2), students with indoor SHS exposure experienced a significant prevalence of probable depressive symptoms $(\mathrm{OR}=1.38$; $95 \% \mathrm{CI} 1.16-1.63$, for SHS in indoor public places; OR $=1.24 ; 95 \%$ CI 1.04-1.47, for SHS in homes; OR = 1.57; 95\% CI 1.29-1.92, for SHS in indoor campuses). When examining the relations by source of exposure, there were similar positive relations for SHS exposure in indoor campuses from smoking teachers (OR $=1.43 ; 95 \% \mathrm{CI} 1.15-1.78)$ and from smoking classmates $(\mathrm{OR}=2.12 ; 95 \% \mathrm{CI} 1.64-2.74)$. We note that the effects of SHS exposure in outdoor campuses cannot be ignored. There was a significant association between SHS exposure in outdoor campuses and probable depressive symptoms (OR $=1.49 ; 95 \% \mathrm{CI} 1.24-1.79)$, and similar positive associations were observed for SHS exposure in outdoor campuses from smoking teachers $(\mathrm{OR}=1.65$; 95\% CI 1.34-2.04) and from smoking classmates (OR $=1.55 ; 95 \%$ CI 1.26-1.91).

Table 2. Relation between binary SHS exposure and probable depressive symptoms.

\begin{tabular}{|c|c|c|c|c|}
\hline SHS Exposure & $n$ & $\begin{array}{c}\text { Probable Depressive } \\
\text { Symptoms }(\%)\end{array}$ & $\begin{array}{l}\text { Unadjusted OR } \\
(95 \% \mathrm{CI})\end{array}$ & $\begin{array}{l}\text { Adjusted OR } \\
(95 \% \mathrm{CI})^{\mathrm{a}}\end{array}$ \\
\hline \multicolumn{5}{|c|}{ SHS in indoor public places } \\
\hline No & 1806 & $428(23.7)$ & 1.00 & 1.00 \\
\hline Yes & 1769 & $630(35.6)$ & $1.78(1.54-2.06)$ & $1.38(1.16-1.63)$ \\
\hline \multicolumn{5}{|l|}{ SHS in homes } \\
\hline No & 2342 & $619(26.4)$ & 1.00 & 1.00 \\
\hline Yes & 1233 & $439(35.6)$ & $1.54(1.33-1.79)$ & $1.24(1.04-1.47)$ \\
\hline \multicolumn{5}{|c|}{ SHS in indoor campuses } \\
\hline No & 2763 & $761(27.5)$ & 1.00 & 1.00 \\
\hline Yes & 812 & 297(36.6) & $1.52(1.29-1.79)$ & $1.57(1.29-1.92)$ \\
\hline \multicolumn{5}{|c|}{ SHS in indoor campuses from smoking teachers } \\
\hline No & 2940 & $837(28.5)$ & 1.00 & 1.00 \\
\hline Yes & 635 & $221(34.8)$ & $1.34(1.12-1.61)$ & $1.43(1.15-1.78)$ \\
\hline
\end{tabular}


Table 2. Cont.

\begin{tabular}{|c|c|c|c|c|}
\hline SHS Exposure & $n$ & $\begin{array}{l}\text { Probable Depressive } \\
\text { Symptoms (\%) }\end{array}$ & $\begin{array}{l}\text { Unadjusted OR } \\
(95 \% \mathrm{CI})\end{array}$ & $\begin{array}{c}\text { Adjusted OR } \\
(95 \% \mathrm{CI})^{\mathrm{a}}\end{array}$ \\
\hline \multicolumn{5}{|c|}{ SHS in indoor campuses from smoking classmates } \\
\hline No & 3149 & $873(27.7)$ & 1.00 & 1.00 \\
\hline Yes & 426 & 185(43.4) & $2.00(1.63-2.46)$ & $2.12(1.64-2.74)$ \\
\hline \multicolumn{5}{|c|}{ SHS in outdoor campuses } \\
\hline No & 2532 & $674(26.6)$ & 1.00 & 1.00 \\
\hline Yes & 1043 & $384(36.8)$ & $1.61(1.38-1.87)$ & $1.49(1.24-1.79)$ \\
\hline \multicolumn{5}{|c|}{ SHS in outdoor campuses from smoking teachers } \\
\hline No & 2917 & $816(28.0)$ & 1.00 & 1.00 \\
\hline Yes & 658 & $242(36.8)$ & $1.50(1.25-1.79)$ & $1.65(1.34-2.04)$ \\
\hline \multicolumn{5}{|c|}{ SHS in outdoor campuses from smoking classmates } \\
\hline No & 2873 & 674(26.6) & 1.00 & 1.00 \\
\hline Yes & 702 & $384(36.8)$ & $1.80(1.52-2.14)$ & $1.55(1.26-1.91)$ \\
\hline
\end{tabular}

$n$, number of participants; SHS, second-hand smoke; OR, odds ratio; CI, confidence interval. a Adjusted for gender, grade, pocket money, parents' education, negative life events, and disease history.

\subsection{Relation between Frequency of SHS Exposure and Probable Depressive Symptoms}

Table 3 reveals the frequency-risk relationships between SHS exposure and probable symptoms. Ordinal frequency of SHS exposure was positively associated with prevalence of probable depressive symptoms in a dose-response manner ( $p$ for linear trend $<0.001$, for SHS in indoor public places, SHS in homes, SHS in indoor campuses, or SHS in outdoor campuses). Similarly, there were monotonically increasing frequency-risk relationships between continuous frequency of SHS exposure and probable depressive symptoms (SHS in indoor public places: OR $=1.74,95 \%$ CI: $1.32-2.29$; SHS in homes: $\mathrm{OR}=1.54,95 \%$ CI: 1.20-1.97; SHS in indoor campuses: OR = 2.50, 95\% CI: 1.76-3.56; SHS in outdoor campuses: $\mathrm{OR}=2.17,95 \% \mathrm{CI}$ : 1.56-3.01). When differentiating these relations for different sources of campus SHS exposure, there were similar frequency-risk relationships for SHS exposure from smoking teachers (SHS in indoor campuses: OR $=2.18,95 \%$ CI: $1.48-3.21$; SHS in outdoor campuses: $\mathrm{OR}=2.52,95 \% \mathrm{CI}: 1.69-3.77$ ) and from smoking classmates (SHS in indoor campuses: $\mathrm{OR}=3.36$, 95\% CI: 2.12-5.31; SHS in outdoor campuses: OR = 2.26, 95\% CI: 1.56-3.29).

Table 3. Relation between frequency of SHS exposure and probable depressive symptoms.

\begin{tabular}{|c|c|c|c|c|}
\hline Frequency of SHS Exposure & $n$ & $\begin{array}{l}\text { Probable Depressive } \\
\text { Symptoms (\%) }\end{array}$ & $\begin{array}{l}\text { Unadjusted OR } \\
(95 \% \mathrm{CI})\end{array}$ & $\begin{array}{l}\text { Adjusted OR } \\
(95 \% \mathrm{CI})^{\mathrm{a}}\end{array}$ \\
\hline SHS in indoor public places ${ }^{b}$ & & & $2.62(2.07-3.31)$ & $1.74(1.32-2.29)$ \\
\hline \multicolumn{5}{|l|}{ SHS in indoor public places } \\
\hline No exposure & 1806 & $428(23.7)$ & 1.00 & 1.00 \\
\hline 1-4 days/week & 1242 & 411(33.1) & $1.59(1.36-1.87)$ & $1.28(1.06-1.53)$ \\
\hline 5-7 days/week & 527 & 219(41.6) & $2.29(1.87-2.81)$ & $1.66(1.30-2.10)$ \\
\hline$p$ for linear trend & & & $<0.001$ & $<0.001$ \\
\hline SHS in homes ${ }^{b}$ & & & $2.02(1.64-2.50)$ & $1.54(1.20-1.97)$ \\
\hline \multicolumn{5}{|l|}{ SHS in homes } \\
\hline No exposure & 2342 & $619(26.4)$ & 1.00 & 1.00 \\
\hline 1-4 days/week & 570 & $176(30.9)$ & $1.24(1.02-1.52)$ & $0.98(0.78-1.24)$ \\
\hline 5-7 days/week & 663 & 263(39.7) & $1.83(1.53-2.19)$ & $1.50(1.22-1.85)$ \\
\hline$p$ for linear trend & & & $<0.001$ & $<0.001$ \\
\hline SHS in indoor campuses ${ }^{b}$ & & & $2.21(1.66-2.94)$ & $2.50(1.76-3.56)$ \\
\hline \multicolumn{5}{|l|}{ SHS in indoor campuses } \\
\hline No exposure & 2763 & $761(27.5)$ & 1.00 & 1.00 \\
\hline 1-4 days/week & 539 & 181(33.6) & 1.33(1.09-1.62) & $1.36(1.08-1.71)$ \\
\hline 5-7 days/week & 273 & $116(42.5)$ & $1.94(1.51-2.51)$ & $2.13(1.56-2.91)$ \\
\hline$p$ for linear trend & & & $<0.001$ & $<0.001$ \\
\hline SHS in indoor campuses from sm & ng teac & & $1.94(1.42-2.66)$ & $2.18(1.48-3.21)$ \\
\hline \multicolumn{5}{|c|}{ SHS in indoor campuses from smoking teachers } \\
\hline No exposure & 2940 & $837(28.5)$ & 1.00 & 1.00 \\
\hline 1-4 days/week & 412 & $130(31.6)$ & $1.16(0.93-1.45)$ & $1.26(0.97-1.63)$ \\
\hline 5-7 days/week & 223 & $91(40.8)$ & $1.73(1.31-2.29)$ & $1.84(1.30-2.58)$ \\
\hline$p$ for linear trend & & & $<0.001$ & $<0.001$ \\
\hline
\end{tabular}


Table 3. Cont.

\begin{tabular}{|c|c|c|c|c|}
\hline Frequency of SHS Exposure & $n$ & $\begin{array}{l}\text { Probable Depressive } \\
\text { Symptoms (\%) }\end{array}$ & $\begin{array}{c}\text { Unadjusted OR } \\
(95 \% \mathrm{CI})\end{array}$ & $\begin{array}{c}\text { Adjusted OR } \\
(95 \% \mathrm{CI})^{\mathrm{a}}\end{array}$ \\
\hline \multicolumn{3}{|c|}{ SHS in indoor campuses from smoking classmates ${ }^{b}$} & $2.74(1.92-3.94)$ & $3.36(2.12-5.31)$ \\
\hline \multicolumn{5}{|c|}{ SHS in indoor campuses from smoking classmates } \\
\hline No exposure & 3149 & $873(27.7)$ & 1.00 & 1.00 \\
\hline 1-4 days/week & 271 & $115(42.4)$ & $1.92(1.49-2.48)$ & $1.89(1.40-2.56)$ \\
\hline 5-7 days/week & 155 & $70(45.2)$ & $2.15(1.55-2.97)$ & $2.67(1.77-4.04)$ \\
\hline$p$ for linear trend & & & $<0.001$ & $<0.001$ \\
\hline SHS in outdoor campuses ${ }^{b}$ & & & $2.20(1.69-2.87)$ & $2.17(1.56-3.01)$ \\
\hline \multicolumn{5}{|l|}{ SHS in outdoor campuses } \\
\hline No exposure & 2532 & 674(26.6) & 1.00 & 1.00 \\
\hline $1-4$ days/week & 704 & $248(35.2)$ & $1.50(1.25-1.79)$ & $1.37(1.11-1.68)$ \\
\hline 5-7 days/week & 339 & $136(40.1)$ & $1.85(1.46-2.33)$ & $1.83(1.38-2.44)$ \\
\hline$p$ for linear trend & & & $<0.001$ & $<0.001$ \\
\hline SHS in outdoor campuses from $s$ & king tea & & $2.21(1.60-3.06)$ & $2.52(1.69-3.77)$ \\
\hline \multicolumn{5}{|c|}{ SHS in outdoor campuses from smoking teachers } \\
\hline No exposure ${ }^{c}$ & 2917 & $816(28.0)$ & 1.00 & 1.00 \\
\hline 1-4 days/week & 456 & 155(34.0) & $1.33(1.07-1.64)$ & $1.48(1.16-1.89)$ \\
\hline 5-7 days/week & 202 & $87(43.1)$ & $1.95(1.46-2.60)$ & $2.16(1.50-3.10)$ \\
\hline$p$ for linear trend & & & $<0.001$ & $<0.001$ \\
\hline SHS in outdoor campuses from $\mathrm{s}$ & king cla & ates $\mathrm{b}$ & $2.34(1.74-3.15)$ & $2.26(1.56-3.29)$ \\
\hline \multicolumn{5}{|c|}{ SHS in outdoor campuses from smoking classmates } \\
\hline No exposure $^{c}$ & 2873 & $777(27.0)$ & 1.00 & 1.00 \\
\hline $1-4$ days/week & 451 & $181(40.1)$ & $1.81(1.47-2.22)$ & $1.42(1.11-1.81)$ \\
\hline 5-7 days/week & 251 & $100(39.8)$ & $1.79(1.37-2.33)$ & $1.86(1.34-2.58)$ \\
\hline$p$ for linear trend & & & $<0.001$ & $<0.001$ \\
\hline
\end{tabular}

$n$, number of participants; SHS, second-hand smoke; OR, odds ratio; CI, confidence interval. ${ }^{a}$ Adjusted for gender, grade, pocket money, parents' education, negative life events, and disease history. ${ }^{\mathrm{b}}$ Logarithmic exposure (days/week) was used in the model.

\subsection{Relation between Binary SHS Exposure and Severe Depressive Symptoms}

Compared with no exposure (Table 4), students with indoor SHS exposure experienced a higher prevalence of severe depressive symptoms (OR $=1.27$; 95\% CI 1.00-1.64, for SHS in homes; OR = 1.66; 95\% CI 1.26-2.18, for SHS in indoor campuses). When examining the relations by source of exposure, similar positive relations were found for SHS exposure in indoor campuses from smoking teachers $(\mathrm{OR}=1.52 ; 95 \% \mathrm{CI} 1.13-2.06)$ and from smoking classmates (OR $=1.99 ; 95 \%$ CI 1.42-2.80). Notably, there was a significant relation between SHS exposure in outdoor campuses and severe depressive symptoms (OR $=1.55 ; 95 \%$ CI 1.20-2.01), and similar relations were observed for SHS exposure in outdoor campuses from smoking teachers $(\mathrm{OR}=1.80 ; 95 \% \mathrm{CI} 1.35-2.40)$ and from smoking classmates $(\mathrm{OR}=1.51 ; 95 \% \mathrm{CI} 1.13-2.03)$.

Table 4. Relation between binary SHS exposure and severe depressive symptoms.

\begin{tabular}{|c|c|c|c|c|}
\hline SHS Exposure & $n$ & $\begin{array}{c}\text { Severe Depressive } \\
\text { Symptoms (\%) }\end{array}$ & $\begin{array}{l}\text { Unadjusted OR } \\
(95 \% \mathrm{CI})\end{array}$ & $\begin{array}{l}\text { Adjusted OR } \\
(95 \% \mathrm{CI})^{\mathrm{a}}\end{array}$ \\
\hline \multicolumn{5}{|c|}{ SHS in indoor public places } \\
\hline No & 1806 & 137(7.6) & 1.00 & 1.00 \\
\hline Yes & 1769 & 206(11.6) & $1.61(1.28-2.01)$ & $1.25(0.98-1.61)$ \\
\hline \multicolumn{5}{|l|}{ SHS in homes } \\
\hline No & 2342 & 195(8.3) & 1.00 & 1.00 \\
\hline Yes & 1233 & $148(12.0)$ & $1.50(1.20-1.88)$ & $1.27(1.00-1.64)$ \\
\hline \multicolumn{5}{|c|}{ SHS in indoor campuses } \\
\hline No & 2763 & $234(8.5)$ & 1.00 & 1.00 \\
\hline Yes & 812 & 109(13.4) & $1.68(1.32-2.13)$ & $1.66(1.26-2.18)$ \\
\hline \multicolumn{5}{|c|}{ SHS in indoor campuses from smoking teachers } \\
\hline No & 2940 & $262(8.9)$ & 1.00 & 1.00 \\
\hline Yes & 635 & $81(12.8)$ & $1.49(1.15-1.95)$ & $1.52(1.13-2.06)$ \\
\hline
\end{tabular}


Table 4. Cont.

\begin{tabular}{|c|c|c|c|c|}
\hline SHS Exposure & $n$ & $\begin{array}{l}\text { Severe Depressive } \\
\text { Symptoms (\%) }\end{array}$ & $\begin{array}{c}\text { Unadjusted OR } \\
(95 \% \mathrm{CI})\end{array}$ & $\begin{array}{c}\text { Adjusted OR } \\
(95 \% \mathrm{CI})^{\mathrm{a}}\end{array}$ \\
\hline \multicolumn{5}{|c|}{ SHS in indoor campuses from smoking classmates } \\
\hline No & 3149 & $274(8.7)$ & 1.00 & 1.00 \\
\hline Yes & 426 & $69(16.2)$ & 2.03(1.52-2.70) & $1.99(1.42-2.80)$ \\
\hline \multicolumn{5}{|c|}{ SHS in outdoor campuses } \\
\hline $\mathrm{No}^{\mathrm{b}}$ & 2532 & $204(8.1)$ & 1.00 & 1.00 \\
\hline Yes & 1043 & 139(13.3) & $1.75(1.40-2.21)$ & $1.55(1.20-2.01)$ \\
\hline \multicolumn{5}{|c|}{ SHS in outdoor campuses from smoking teachers } \\
\hline $\mathrm{No}^{\mathrm{b}}$ & 2917 & $250(8.6)$ & 1.00 & 1.00 \\
\hline Yes & 658 & 93(14.1) & $1.76(1.36-2.27)$ & $1.80(1.35-2.40)$ \\
\hline \multicolumn{5}{|c|}{ SHS in outdoor campuses from smoking classmates } \\
\hline No & 2873 & $204(8.1)$ & 1.00 & 1.00 \\
\hline Yes & 702 & $139(13.3)$ & $1.80(1.40-2.31)$ & $1.51(1.13-2.03)$ \\
\hline
\end{tabular}

n, number of participants; SHS, second-hand smoke; OR, odds ratio; CI, confidence interval. ${ }^{\text {a }}$ Adjusted for gender, grade, pocket money, parents' education, negative life events, and disease history.

\subsection{Relation between Frequency of SHS Exposure and Severe Depressive Symptoms}

Table 5 indicates the frequency-risk relationships between SHS exposure and severe depressive symptoms. Ordinal frequency of indoor SHS exposure was significantly associated with severe depressive symptoms in a dose-response manner (SHS in indoor public places: $p$ for linear trend = 0.036; SHS in homes: $p$ for linear trend $=0.016$; SHS in indoor campuses: $p$ for linear trend $<0.001$; SHS in outdoor campuses: $p$ for linear trend $=0.001$ ). Additionally, there were increasing frequency-risk relationships between continuous frequency of SHS exposure and severe depressive symptoms (SHS in indoor public places: $\mathrm{OR}=1.72,95 \% \mathrm{CI}$ : $1.17-2.55$; $\mathrm{SHS}$ in homes: $\mathrm{OR}=1.51,95 \% \mathrm{CI}$ : $1.06-2.16$; SHS in indoor campuses: OR $=2.64,95 \%$ CI: $1.67-4.16$; SHS in outdoor campuses: OR $=2.21,95 \%$ CI: 1.43-3.42). When differentiating these relations for different sources of campus SHS exposure, there were similar dose-response relationships for SHS exposure from smoking teachers (SHS in indoor campuses: $\mathrm{OR}=2.29,95 \%$ CI: 1.40-3.77; SHS in outdoor campuses: OR $=2.76,95 \%$ CI: 1.67-4.54) and from smoking classmates (SHS in indoor campuses: OR $=3.04,95 \%$ CI: 1.71-5.42; SHS in outdoor campuses: $\mathrm{OR}=1.85,95 \%$ CI: $1.12-3.07)$.

Table 5. Relation between frequency of SHS exposure and severe depressive symptoms.

\begin{tabular}{|c|c|c|c|c|}
\hline Frequency of SHS Exposure & $n$ & $\begin{array}{l}\text { Severe Depressive } \\
\text { Symptoms (\%) }\end{array}$ & $\begin{array}{l}\text { Unadjusted OR } \\
(95 \% \mathrm{CI})\end{array}$ & $\begin{array}{l}\text { Adjusted OR } \\
(95 \% \mathrm{CI})^{\mathrm{a}}\end{array}$ \\
\hline SHS in indoor public places ${ }^{b}$ & & & $2.52(1.79-3.55)$ & $1.72(1.17-2.55)$ \\
\hline \multicolumn{5}{|l|}{ SHS in indoor public places } \\
\hline No exposure & 1806 & 137(7.6) & 1.00 & 1.00 \\
\hline $1-4$ days/week & 1242 & $128(10.3)$ & $1.40(1.09-1.80)$ & $1.13(0.86-1.49)$ \\
\hline 5-7 days/week & 527 & $78(14.8)$ & $2.12(1.57-2.85)$ & $1.44(1.02-2.02)$ \\
\hline$p$ for linear trend & & & $<0.001$ & 0.036 \\
\hline SHS in homes ${ }^{b}$ & & & $1.87(1.36-2.56)$ & $1.51(1.06-2.16)$ \\
\hline \multicolumn{5}{|l|}{ SHS in homes } \\
\hline No exposure & 2342 & 195(8.3) & 1.00 & 1.00 \\
\hline 1-4 days/week & 570 & $59(10.4)$ & $1.27(0.94-1.73)$ & $1.09(0.77-1.52)$ \\
\hline 5-7 days/week & 663 & $89(13.4)$ & $1.70(1.31-2.23)$ & $1.45(1.07-1.95)$ \\
\hline$p$ for linear trend & & & $<0.001$ & 0.016 \\
\hline SHS in indoor campuses ${ }^{b}$ & & & $2.61(1.76-3.86)$ & $2.64(1.67-4.16)$ \\
\hline \multicolumn{5}{|l|}{ SHS in indoor campuses } \\
\hline No exposure & 2763 & $234(8.5)$ & 1.00 & 1.00 \\
\hline 1-4 days/week & 539 & $63(11.7)$ & $1.43(1.07-1.92)$ & $1.46(1.05-2.03)$ \\
\hline 5-7 days/week & 273 & $46(16.9)$ & $2.19(1.55-3.09)$ & $2.10(1.40-3.12)$ \\
\hline \multicolumn{3}{|c|}{$p$ for linear trend } & $<0.001$ & $<0.001$ \\
\hline
\end{tabular}


Table 5. Cont.

\begin{tabular}{|c|c|c|c|c|}
\hline Frequency of SHS Exposure & $n$ & $\begin{array}{l}\text { Severe Depressive } \\
\text { Symptoms (\%) }\end{array}$ & $\begin{array}{l}\text { Unadjusted OR } \\
(95 \% \mathrm{CI})\end{array}$ & $\begin{array}{l}\text { Adjusted OR } \\
(95 \% \mathrm{CI})^{\mathrm{a}}\end{array}$ \\
\hline \multicolumn{3}{|c|}{ SHS in indoor campuses from smoking teachers ${ }^{b}$} & $2.39(1.55-3.69)$ & $2.29(1.40-3.77)$ \\
\hline \multicolumn{5}{|c|}{ SHS in indoor campuses from smoking teachers } \\
\hline No exposure & 2940 & $262(8.9)$ & 1.00 & 1.00 \\
\hline $1-4$ days/week & 412 & $42(10.2)$ & $1.16(0.82-1.64)$ & $1.27(0.87-1.85)$ \\
\hline 5-7 days/week & 223 & $39(17.5)$ & $2.17(1.50-3.13)$ & $2.01(1.31-3.09)$ \\
\hline$p$ for linear trend & & & $<0.001$ & 0.001 \\
\hline SHS in indoor campuses from & king c & smates $^{b}$ & $2.83(1.76-4.56)$ & $3.04(1.71-5.42)$ \\
\hline \multicolumn{5}{|c|}{ SHS in indoor campuses from smoking classmates } \\
\hline No exposure & 3149 & $274(8.7)$ & 1.00 & 1.00 \\
\hline 1-4 days/week & 271 & $43(15.9)$ & $1.98(1.40-2.80)$ & $1.89(1.27-2.82)$ \\
\hline 5-7 days/week & 155 & $26(16.8)$ & $2.11(1.36-3.28)$ & $2.20(1.30-3.72)$ \\
\hline$p$ for linear trend & & & 0.001 & 0.003 \\
\hline SHS in outdoor campuses ${ }^{b}$ & & & $2.57(1.77-3.71)$ & $2.21(1.43-3.42)$ \\
\hline \multicolumn{5}{|l|}{ SHS in outdoor campuses } \\
\hline No exposure & 2532 & 204(8.1) & 1.00 & 1.00 \\
\hline $1-4$ days/week & 704 & $84(11.9)$ & $1.55(1.18-2.02)$ & $1.40(1.04-1.88)$ \\
\hline 5-7 days/week & 339 & $55(16.2)$ & $2.21(1.60-3.05)$ & $1.94(1.32-2.83)$ \\
\hline$p$ for linear trend & & & $<0.001$ & 0.001 \\
\hline SHS in outdoor campuses from & noking & chers ${ }^{b}$ & $2.87(1.86-4.41)$ & $2.76(1.67-4.54)$ \\
\hline \multicolumn{5}{|c|}{ SHS in outdoor campuses from smoking teachers } \\
\hline No exposure & 2917 & $250(8.6)$ & 1.00 & 1.00 \\
\hline $1-4$ days/week & 456 & $55(12.1)$ & $1.46(1.07-2.00)$ & $1.57(1.12-2.21)$ \\
\hline 5-7 days/week & 202 & $38(18.8)$ & $2.47(1.70-3.60)$ & $2.36(1.52-3.66)$ \\
\hline$p$ for linear trend & & & $<0.001$ & $<0.001$ \\
\hline SHS in outdoor campuses from & noking & issmates ${ }^{b}$ & $2.16(1.42-3.29)$ & $1.85(1.12-3.07)$ \\
\hline \multicolumn{5}{|c|}{ SHS in outdoor campuses from smoking classmates } \\
\hline No exposure & 2873 & $243(8.5)$ & 1.00 & 1.00 \\
\hline 1-4 days/week & 451 & 63(14.0) & $1.76(1.31-2.36)$ & 1.43(1.02-1.99) \\
\hline 5-7 days/week & 251 & $37(14.7)$ & $1.87(1.29-2.72)$ & $1.72(1.10-2.68)$ \\
\hline$p$ for linear trend & & & 0.001 & 0.016 \\
\hline
\end{tabular}

$n$, number of participants; SHS, second-hand smoke; OR, odds ratio; CI, confidence interval. ${ }^{a}$ Adjusted for gender, grade, pocket money, parents' education, negative life events, and disease history. ${ }^{\mathrm{b}}$ Logarithmic exposure (days/week) was used in the model.

\section{Discussion}

This study builds on previous literature by addressing the potential frequency-risk relationships between SHS exposure and depressive symptoms among adolescents and differentiating these associations in setting-specific exposure and severity-specific outcomes. We found that there were monotonically increasing frequency-risk relationships between setting-specific (public places, homes, or campuses) SHS exposure and severity-specific (probable or severe) depressive symptoms. When examining these relations by source of exposure, there were similar positive and dose-response relationships for SHS exposure from smoking teachers and from smoking classmates.

Association between SHS exposure and depressive symptoms has been reported previously, but the findings are inconsistent [18,31-33]. The latest study of Chinese middle-aged women found that SHS exposure in homes was positively associated with depressive symptoms, but no association was observed for SHS exposure in indoor public places [32]. On the contrary, the study of Japanese workers indicated that SHS exposure at work was related with higher rates of depressive symptoms, but no relation was found for SHS exposure at home [18]. Additionally, the latest study on Chinese middle-aged women revealed a significant dose-response relationship between SHS exposure in homes and depressive symptoms [32], but the Korea National Health and Nutrition Examination Survey on men observed no dose-response association for home or workplace SHS exposure [34]. To date, only two studies have explored this relation in a population of adolescents, indicating that there was a significant relation between SHS exposure and depression or depressive symptoms [20,21]. However, it was unclear whether there is a dose-response relationship between SHS exposure and depressive symptoms among adolescents, and the potential relationships for setting-specific 
exposure and severity-specific outcomes were also unclear. Therefore, we aimed to assess the potential frequency-risk relationship among adolescents and differentiate this relationship in setting-specific (public places or homes) exposure and severity-specific (probable or severe) outcomes to make exposure and outcomes clearer. We found that there were frequency-risk relationships between setting-specific SHS exposure and severity-specific depressive symptoms. These findings point out the urgent need for comprehensive smoke-free legislation covering all public places and workplaces in Guangzhou to protect the public from SHS hazards, as called for in Article 8 of the Framework Convention on Tobacco Control. The above findings also suggest that the setting-specific associations between SHS exposure and depressive symptoms may differ in populations investigated, which may be due to differences in dose, frequency, and duration of SHS exposure in specific settings (such as homes, public places, and workplaces).

We note that much attention has been focused on SHS exposure in public places and in homes among adolescents, but there are still few studies regarding SHS exposure in indoor and outdoor campuses. Campus smoking bans were implemented in Guangzhou on 1 September 2010, and a full smoking ban (100\% smoke-free) covers indoor and outdoor campuses. However, SHS exposure in indoor $(22.7 \%)$ or outdoor $(29.2 \%)$ campuses was still at a high level, which is consistent with results from other countries [35-37]. These findings suggest poor compliance with the full smoke-free ban in campuses. A few published studies have revealed that SHS exposure may be a risk factor of depressive symptoms, but only focused on SHS exposure in homes or in public places [18,21,32-34]. Of concern is that the potential relationship between SHS exposure in campuses and depressive symptoms is still unclear. Our study found positive relations and monotonically increasing dose-response relationships between setting-specific (indoor or outdoor campuses) SHS exposure and severity-specific (probable or severe) depressive symptoms. When differentiating these relationships for different sources of campus SHS exposure, there were similar dose-response relationships found for SHS exposure in campuses from smoking teachers or from smoking classmates. These findings provide more evidence for the potential influence of campus SHS exposure on depressive symptoms among adolescents, and support growing concern about SHS exposure in both indoor and outdoor campuses. These findings also indicate that the Guangzhou government needs to take measures to ensure the effective implementation of a full smoking ban in both indoor and outdoor campuses.

Although the nature of the relationship between SHS exposure and depression is uncertain, there are some possible mechanisms that may explain its relationship. Firstly, two biological mechanisms may explain the effects of SHS exposure on depression. One is the neurobiological mechanism observed in smokers and nonsmokers with SHS exposure. Several animal studies have revealed that nicotine exposure has acute and long-term effects on the dopamine system, which may lead to long-term imbalances in dopamine transport [9]. Lower levels of dopamine result from prolonged exposure to SHS, and have also been related to increased risk of negative mood or depression [10]. Another biological mechanism that relates SHS exposure to depressive symptoms is chronic inflammation [11,12]. Many studies have proposed that inflammatory cytokines induce the indoleamine 2,3-dioxygenase, which limits tryptophan and serotonin transport and may thus cause depression [13]. Secondly, there is increasing evidence suggesting that regular SHS exposure may be an indicator of a stressful working and living environment, and such chronic stress may be associated with the worsening of depressive symptoms through impaired neuroplasticity mechanisms and abnormal neurotrophic factors levels [8]. For example, chronic stress and major depression are associated with structural brain changes such as a loss of dendritic spines and synapses, reduced dendritic arborization, and diminished glial cells in the hippocampus [7].

Depression is one of the most common mental disorders and is considered a major public health problem. Moreover, adolescent depression is an antecedent of many adverse outcomes in adulthood, and globally imposes a significant economic burden not only on individuals with the condition, but also on their families and communities [38]. The present study found that $29.9 \%$ of the participants had probable depressive symptoms, which is similar to previous findings from other 
Asian countries (27.1\% for university students in Turkey and 31.4\% for Korean adolescents) [20,39]. However, the prevalence of depressive symptoms was low in adolescents in the United States $(8.2 \%$ of females and $6.8 \%$ of males) [40]. These variations in the reported rates of depressive symptoms could be due, at least in part, to differences in populations investigated and in instruments for assessing depressive symptoms. Although there is no absolute consensus on which cutoff score is the best for discriminating clinically depressed persons from nondepressed persons, some authors have suggested that higher cutoff scores may reduce false-positive rates and improve agreement [41]. To make depressive symptoms more accurate, our study also used a higher cutoff point for depressive symptoms (that is, CES-D score $\geq 25$ for severe depressive symptoms), on which has been placed particular emphasis in Asian countries and in some Hispanic ethnic groups [41-43]. Notably, the prevalence of severe depressive symptoms among adolescents was $9.4 \%$, which was slightly higher than in Korean adults $(8.7 \%)$ but lower than in Chinese college freshmen $(17.6 \%)[26,44]$. Note that screening is an important step in identifying depression in adolescents and securing appropriate treatment for adolescents experiencing severe depressive symptoms. There are a variety of depression screening tools available, including the Beck Depression Inventory-II, Patient Health Questionnaire-Adolescent Version, and Children's Depression Inventory [45]. These tools take approximately 5 to $10 \mathrm{~min}$ to complete, including written assessments completed by the parent or adolescent and interview-style assessments administered by nurse practitioners.

There are some limitations that should be considered when interpreting the results. Firstly, the study design is cross-sectional, in which both exposure and outcomes are measured the same time; therefore, we can only describe a potential association between SHS exposure and depressive symptoms, not a causal conclusion. Owing to the cross-sectional design of this study, it is possible that participants with depressive symptoms are more likely to report exposure than those without symptoms. Therefore, results from this study need to be confirmed in a longitudinal study. Secondly, smoking in previous studies was defined as "smoking 1 day or more within the past 30 days" or "smoking 1 cigarette or more within the past 7 days" [46,47]. However, smoking in this study was defined as "a history of smoking more than 100 cigarettes during the participant's lifetime", which is consistent with other previous studies but may underestimate the true prevalence of smokers [28-30]. Thirdly, SHS exposure in this study is based on self-reporting and has no biochemical measures (e.g., serum nicotine and cotinine). However, self-reported data obtained from population-based surveys was generally valid, apart from when there is a high demand for abstinence [48]. Additionally, self-reported SHS exposure allows the prevalence and frequency of exposure to be assessed, so we can explore the frequency-risk relationships and differentiate SHS exposure in different venues to explore setting-specific relations.

\section{Conclusions}

In conclusion, this study additionally contributes to the literature by exploring the frequency-risk relationships between SHS exposure and depressive symptoms in setting-specific exposure and severity-specific outcomes. We found that there were monotonically increasing frequency-risk relationships between setting-specific (public places, homes, or campuses) SHS exposure and severity-specific (probable or severe) depressive symptoms. These findings highlight the need for further longitudinal studies to establish the causal relationship and the need for smoke-free environments to protect the health of adolescents.

Author Contributions: J.H., B.X., G.L. and X.Y. conceived and designed the study; J.H., B.X., D.G., T.J., W.H., G.L. and X.Y. collected the data; J.H. and X.Y. analyzed the data and wrote the paper; all co-authors participated in the revising of the manuscript and approved the version submitted for publication.

Funding: This work was supported by the Science and Technology Project of Guangzhou Health Bureau (No. 2014-1633). The funders played no role in the study design or in the data collection, analysis, and interpretation.

Conflicts of Interest: The authors declare that they have no conflict of interest regarding the publication of this paper. 


\section{References}

1. Warren, G.W.; Alberg, A.J.; Kraft, A.S.; Cummings, K.M. The 2014 Surgeon General's report: "The health consequences of smoking-50 years of progress": A paradigm shift in cancer care. Cancer 2014, 120, 1914-1916. [CrossRef] [PubMed]

2. Oberg, M.; Jaakkola, M.S.; Woodward, A.; Peruga, A.; Pruss-Ustun, A. Worldwide burden of disease from exposure to second-hand smoke: A retrospective analysis of data from 192 countries. Lancet 2011, 377, 139-146. [CrossRef]

3. Office of the Leading Group for Implementation of the Framework Convention on Tobacco Control. China tobacco control report 2007. Chin. J. Health Educ. 2008, 24, 934-939.

4. Warren, C.W.; Jones, N.R.; Peruga, A.; Chauvin, J.; Baptiste, J.P.; Costa de Silva, V.; elAwa, F.; Tsouros, A.; Rahman, K.; Fishburn, B.; et al. Global youth tobacco surveillance, 2000-2007. MMWR Surveill. Summ. 2008, 57, 1-28. [PubMed]

5. Edwards, A.C.; Kendler, K.S. A twin study of depression and nicotine dependence: Shared liability or causal relationship? J. Affect. Disord. 2012, 142, 90-97. [CrossRef] [PubMed]

6. Menezes, A.M.; Murray, J.; László, M.; Wehrmeister, F.C.; Hallal, P.C.; Gonçalves, H.; Assunção, M.C.; Menezes, C.B.; Barros, F.C. Happiness and depression in adolescence after maternal smoking during pregnancyirth cohort study. PLoS ONE 2013, 8, e80370. [CrossRef] [PubMed]

7. Serafini, G.; Hayley, S.; Pompili, M.; Dwivedi, Y.; Brahmachari, G.; Girardi, P.; Amore, M. Hippocampal neurogenesis, neurotrophic factors and depression: Possible therapeutic targets? CNS Neurol. Disord. Drug Targets 2014, 13, 1708-1721. [CrossRef] [PubMed]

8. Hammen, C. Stress and depression. Annu. Rev. Clin. Psychol. 2005, 1, 293-319. [CrossRef] [PubMed]

9. Danielson, K.; Putt, F.; Truman, P.; Kivell, B.M. The effects of nicotine and tobacco particulate matter on dopamine uptake in the rat brain. Synapse 2014, 68, 45-60. [CrossRef] [PubMed]

10. Iñiguez, S.D.; Warren, B.L.; Parise, E.M.; Alcantara, L.F.; Schuh, B.; Maffeo, M.L.; Manojlovic, Z.; Bolaños-Guzmán, C.A. Nicotine exposure during adolescence induces a depression-like state in adulthood. Neuropsychopharmacology 2009, 34, 1609-1624. [CrossRef] [PubMed]

11. Adams, T.; Wan, E.; Wei, Y.; Wahab, R.; Castagna, F.; Wang, G.; Emin, M.; Russo, C.; Homma, S.; Le Jemtel, T.H.; et al. Secondhand Smoking Is Associated With Vascular Inflammation. Chest 2015, 148, 112-119. [CrossRef] [PubMed]

12. Jefferis, B.J.; Lowe, G.D.; Welsh, P.; Rumley, A.; Lawlor, D.A.; Ebrahim, S.; Carson, C.; Doig, M.; Feyerabend, C.; McMeekin, L.; et al. Secondhand smoke (SHS) exposure is associated with circulating markers of inflammation and endothelial function in adult men and women. Atherosclerosis 2010, 208, 550-556. [CrossRef] [PubMed]

13. Dantzer, R.; O'Connor, J.C.; Lawson, M.A.; Kelley, K.W. Inflammation-associated depressionrom serotonin to kynurenine. Psychoneuroendocrinology 2011, 36, 426-436. [CrossRef] [PubMed]

14. Gim, W.; Yoo, J.H.; Shin, J.Y.; Goo, A.J. Relationship between Secondhand Smoking with Depressive Symptom and Suicidal Ideation in Korean Non-Smoker Adults: The Korean National Health and Nutrition Examination Survey 2010-2012. Korean J. Fam. Med. 2016, 37, 97-104. [CrossRef] [PubMed]

15. Kim, N.H.; Choi, H.; Kim, N.R.; Shim, J.S.; Kim, H.C. Secondhand smoke exposure and mental health problems in Korean adults. Epidemiol. Health 2016, 38, e2016009. [CrossRef]

16. Zeng, Y.N.; Li, Y.M. Secondhand smoke exposure and mental health in adults: A meta-analysis of crosssectional studies. Soc. Psychiatry Psychiatr. Epidemiol. 2016, 51, 1339-1348. [CrossRef] [PubMed]

17. Bandiera, F.C.; Arheart, K.L.; Caban-Martinez, A.J.; Fleming, L.E.; McCollister, K.; Dietz, N.A.; Leblanc, W.G.; Davila, E.P.; Lewis, J.E.; Serdar, B.; et al. Secondhand smoke exposure and depressive symptoms. Psychosom. Med. 2010, 72, 68-72. [CrossRef] [PubMed]

18. Nakata, A.; Takahashi, M.; Ikeda, T.; Hojou, M.; Nigam, J.A.; Swanson, N.G. Active and passive smoking and depression among Japanese workers. Prev. Med. 2008, 46, 451-456. [CrossRef] [PubMed]

19. Bot, M.; Vink, J.M.; Willemsen, G.; Smit, J.H.; Neuteboom, J.; Kluft, C.; Boomsma, D.I.; Penninx, B.W. Exposure to secondhand smoke and depression and anxiety: A report from two studies in the Netherlands. J. Psychosom. Res. 2013, 75, 431-436. [CrossRef] [PubMed]

20. Kim, N.H.; Park, J.H.; Choi, D.P.; Lee, J.Y.; Kim, H.C. Secondhand Smoke Exposure and Depressive Symptoms among Korean Adolescents: JS High School Study. PLoS ONE 2016, 11, e0168754. [CrossRef] [PubMed] 
21. Lee, K.J. Current smoking and secondhand smoke exposure and depression among Korean adolescents: Analysis of a national cross-sectional survey. BMJ Open 2014, 4, e003734. [CrossRef] [PubMed]

22. Radloff, L.S. The CES-D Scale: A Self-Report Depression Scale for Research in the General Population. Appl. Psychol. Meas. 1977, 1, 385-401. [CrossRef]

23. Van Dijk, M.L.; Savelberg, H.H.C.M.; Verboon, P.; Kirschner, P.A.; De Groot, R.H.M. Decline in physical activity during adolescence is not associated with changes in mental health. BMC Public Health 2016, 16, 300. [CrossRef] [PubMed]

24. Xu, Y.; Qi, J.; Yang, Y.; Wen, X. The contribution of lifestyle factors to depressive symptoms: A cross-sectional study in Chinese college students. Psychiatry Res. 2016, 245, 243-249. [CrossRef] [PubMed]

25. Huang, J.; Wen, G.; Yang, W.; Yao, Z.; Wu, C.; Ye, X. The association between second-hand smoke exposure and depressive symptoms among pregnant women. Psychiatry Res. 2017, 256, 469-474. [CrossRef] [PubMed]

26. Cho, M.J.; Nam, J.J.; Suh, G.H. Prevalence of symptoms of depression in a nationwide sample of Korean adults. Psychiatry Res. 1998, 81, 341-352. [CrossRef]

27. Chinese Center For Control And Prevention. Global Adult Tobacco Survey (GATS): China 2010 Country Report; China Three Gorges Press: Beijing, China, 2011.

28. Selya, A.S.; Dierker, L.; Rose, J.S.; Hedeker, D.; Mermelstein, R.J. Early-Emerging Nicotine Dependence Has Lasting and Time-Varying Effects on Adolescent Smoking Behavior. Prev. Sci. 2016, 17, 743-750. [CrossRef] [PubMed]

29. Selya, A.S.; Dierker, L.C.; Rose, J.S.; Hedeker, D.; Tan, X.; Li, R.; Mermelstein, R.J. Time-varying effects of smoking quantity and nicotine dependence on adolescent smoking regularity. Drug Alcohol. Depend. 2013, 128, 230-237. [CrossRef] [PubMed]

30. Chen, J.M.; Hwang, B.F.; Chen, Y.C.; Lee, Y.L. Active smoking, environmental tobacco smoke and bronchitic symptoms among adolescents in Taiwan prospective cohort study. Prev. Med. 2014, 65, 116-121. [CrossRef] [PubMed]

31. Kim, N.H.; Kim, H.C.; Lee, J.Y.; Lee, J.M.; Suh, I. Association between environmental tobacco smoke and depression among Korean women. BMJ Open 2015, 5, e007131. [CrossRef] [PubMed]

32. Ye, X.; Li, L.; Gao, Y.; Zhou, S.; Yang, Y.; Chen, S. Dose-response relations between second-hand smoke exposure and depressive symptoms among middle-aged women. Psychiatry Res. 2015, 229, 533-538. [CrossRef] [PubMed]

33. Tan, S.; Courtney, L.P.; El-Mohandes, A.A.; Gantz, M.G.; Blake, S.M.; Thornberry, J.; El-Khorazaty, M.N.; Perry, D.; Kiely, M.; et al. Relationships between self-reported smoking, household environmental tobacco smoke exposure and depressive symptoms in a pregnant minority population. Matern. Child Health J. 2011, 15 (Suppl. 1), S65-S74. [CrossRef] [PubMed]

34. Jung, S.J.; Shin, A.; Kang, D. Active smoking and exposure to secondhand smoke and their relationship to depressive symptoms in the Korea national health and nutrition examination survey (KNHANES). BMC Public Health 2015, 15, 1053. [CrossRef] [PubMed]

35. Thrasher, J.F.; Swayampakala, K.; Arillo-Santillán, E.; Sebrié, E.; Walsemann, K.M.; Bottai, M. Differential impact of local and federal smoke-free legislation in Mexico: A longitudinal study among adult smokers. Salud Publica Mex. 2010, 52 (Suppl. 2), S244-S253. [CrossRef] [PubMed]

36. Edwards, R.; Thomson, G.; Wilson, N.; Waa, A.; Bullen, C.; O’Dea, D.; Gifford, H.; Glover, M.; Laugesen, M.; Woodward, A. After the smoke has clearedvaluation of the impact of a new national smoke-free law in New Zealand. Tob. Control 2008, 17, e2. [CrossRef] [PubMed]

37. Nagelhout, G.E.; de Vries, H.; Boudreau, C.; Allwright, S.; McNeill, A.; van den Putte, B.; Fong, G.T.; Willemsen, M.C. Comparative impact of smoke-free legislation on smoking cessation in three European countries. Eur. J. Public Health 2012, 22 (Suppl. 1), 4-9. [CrossRef] [PubMed]

38. Fergusson, D.M.; Woodward, L.J. Mental health, educational, and social role outcomes of adolescents with depression. Arch. Gen. Psychiatry 2002, 59, 225-231. [CrossRef] [PubMed]

39. Bayram, N.; Bilgel, N. The prevalence and socio-demographic correlations of depression, anxiety and stress among a group of university students. Soc. Psychiatry Psychiatr. Epidemiol. 2008, 43, 667-672. [CrossRef] [PubMed]

40. Needham, B.L.; Crosnoe, R. Overweight status and depressive symptoms during adolescence. J. Adolesc. Health 2005, 36, 48-55. [CrossRef] [PubMed] 
41. Cho, M.J.; Mościcki, E.K.; Narrow, W.E.; Rae, D.S.; Locke, B.Z.; Regier, D.A. Concordance between two measures of depression in the Hispanic Health and Nutrition Examination Survey. Soc. Psychiatry Psychiatr. Epidemiol. 1993, 28, 156-163. [CrossRef] [PubMed]

42. Iwata, N.; Saito, K.; Roberts, R.E. Responses to a self-administered depression scale among younger adolescents in Japan. Psychiatry Res. 1994, 53, 275-287. [CrossRef]

43. Cho, M.J.; Kim, K.H. Use of the Center for Epidemiologic Studies Depression (CES-D) Scale in Korea. J. Nerv. Ment. Dis. 1998, 186, 304-310. [CrossRef] [PubMed]

44. Song, Y.; Huang, Y.; Liu, D.; Kwan, J.S.; Zhang, F.; Sham, P.C.; Tang, S.W. Depression in collegeepressive symptoms and personality factors in Beijing and Hong Kong college freshmen. Compr. Psychiatry 2008, 49, 496-502. [CrossRef] [PubMed]

45. Hamrin, V.; Antenucci, M.; Magorno, M. Evaluation and management of pediatric and adolescent depression. Nurse Pract. 2012, 37, 22-30. [CrossRef] [PubMed]

46. Itanyi, I.U.; Onwasigwe, C.N.; McIntosh, S.; Bruno, T.; Ossip, D.; Nwobi, E.A.; Onoka, C.A.; Ezeanolue, E.E. Disparities in tobacco use by adolescents in southeast, Nigeria using Global Youth Tobacco Survey (GYTS) approach. BMC Public Health 2018, 18, 317. [CrossRef] [PubMed]

47. Mak, K.K.; Ho, R.C.; Day, J.R. The Associations of Asthma Symptoms with Active and Passive Smoking in Hong Kong Adolescents. Respir. Care 2012, 57, 1398-1404. [CrossRef] [PubMed]

48. Jung-Choi, K.H.; Khang, Y.H.; Cho, H.J. Hidden female smokers in Asia comparison of self-reported with cotinine-verified smoking prevalence rates in representative national data from an Asian population. Tob. Control 2012, 21, 536-542. [CrossRef] [PubMed]

(C) 2018 by the authors. Licensee MDPI, Basel, Switzerland. This article is an open access article distributed under the terms and conditions of the Creative Commons Attribution (CC BY) license (http://creativecommons.org/licenses/by/4.0/). 